\title{
Cooling of a One-Dimensional Bose Gas
}

\author{
B. Rauer, ${ }^{1, *}$ P. Grišins, ${ }^{1, \dagger}$ I. E. Mazets, ${ }^{1,2}$ T. Schweigler, ${ }^{1}$ W. Rohringer, ${ }^{1}$ \\ R. Geiger, ${ }^{1, \$}$ T. Langen, ${ }^{1, \S}$ and J. Schmiedmayer ${ }^{1}$ \\ ${ }^{1}$ Vienna Center for Quantum Science and Technology, Atominstitut, TU Wien, Stadionallee 2, 1020 Vienna, Austria \\ ${ }^{2}$ Wolfgang Pauli Institute, 1090 Vienna, Austria \\ (Received 11 June 2015; published 22 January 2016)
}

\begin{abstract}
We experimentally study the dynamics of a degenerate one-dimensional Bose gas that is subject to a continuous outcoupling of atoms. Although standard evaporative cooling is rendered ineffective by the absence of thermalizing collisions in this system, we observe substantial cooling. This cooling proceeds through homogeneous particle dissipation and many-body dephasing, enabling the preparation of otherwise unexpectedly low temperatures. Our observations establish a scaling relation between temperature and particle number, and provide insights into equilibration in the quantum world.
\end{abstract}

DOI: 10.1103/PhysRevLett.116.030402

Introduction.-Long coherence times, the tunability of many parameters, and the ability to precisely probe and manipulate their quantum state make ultracold atomic gases a very promising and versatile tool to study the physics of quantum many-body systems [1]. The standard technique to reach the necessary ultracold temperatures in these systems is evaporative cooling [2-4], a fundamental process present in many physical systems from hot liquids to stellar clusters. It relies on the selective removal of the most energetic particles from a trapped gas and the subsequent rethermalization of the gas to a lower temperature through elastic collisions. For efficient cooling this cycle is repeated continuously, increasing the phase-space density of a gas at the cost of reducing the total number of atoms.

Intuitively, systems that do not thermalize cannot be cooled through particle dissipation. In this Letter we demonstrate that this notion is incomplete by studying the dissipative dynamics of a one-dimensional Bose gas with contact interactions. We observe a substantial decrease in temperature as a result of homogeneous particle dissipation, reaching temperatures far below $\hbar \omega_{\perp}$, the energy characterizing the transverse confinement. In this deep 1D regime thermalization is strongly suppressed [5,6]: Thermalizing two-body collisions are frozen out [7] and three-body collisions $[7,8]$ or phonon-phonon scattering [9-11] can be neglected on our time scales. We find that the observed cooling can be modeled as a continuous density reduction extracting energy from the density quadrature of the free phononic excitations. Together with a continuous many-body dephasing, this reduces the occupation number of each phonon mode and leads to a colder system.

Published by the American Physical Society under the terms of the Creative Commons Attribution 3.0 License. Further distribution of this work must maintain attribution to the author(s) and the published article's title, journal citation, and DOI.
Experiment.-Our experimental system is a 1D Bose gas of ${ }^{87} \mathrm{Rb}$ atoms prepared in the anisotropic magnetic trapping potential of an atom chip [12]. Initially, a precooled three-dimensional cloud of thermal atoms, prepared in the $\left|F, m_{F}\right\rangle=|2,2\rangle$ ground state, is loaded into the trapping potential. The gas is then cooled through the condensate transition and into the $1 \mathrm{D}$ regime by conventional evaporative cooling. The evaporation is realized through a weak driving of energy-selective radio-frequency (rf) transitions to untrapped Zeeman states [13]. The cooling ramp of the rf is performed over $1.6 \mathrm{~s}$, with the final part being particularly slow to minimize collective excitations in the gas. The 1D regime is reached when both the temperature and the chemical potential drop below $\hbar \omega_{\perp}$, the energy spacing between the ground state and the first excited state of the transverse confinement. At this point the condensate typically consists of 7000-10000 atoms at an initial temperature $T$ of $30-100 \mathrm{nK}$. The resulting macroscopic quantum system is a quasicondensate described by a macroscopic wave function with a fluctuating phase [14].

This trapped 1D quasicondensate is the starting point of our experiments. To investigate the effects of particle dissipation, we monitor the further evolution of the system under continuous driving of transitions to untrapped states. The outcoupling rate is tuned in such a way that atoms are removed slowly from the quantum degenerate gas to avoid collective excitations. In contrast to conventional evaporative cooling the atoms are not outcoupled energy selectively but at a nearly homogeneous rate. This is experimentally demonstrated in Fig. 1(a) by extracting a visible fraction of the gas using a single outcoupling pulse and analyzing its relation to the source cloud. The outcoupled atoms show the same average density profile as well as the same density speckle patterns, indicating a coherent and homogeneous outcoupling process.

Information about the system is extracted through absorption imaging in time of flight, transversal to the 


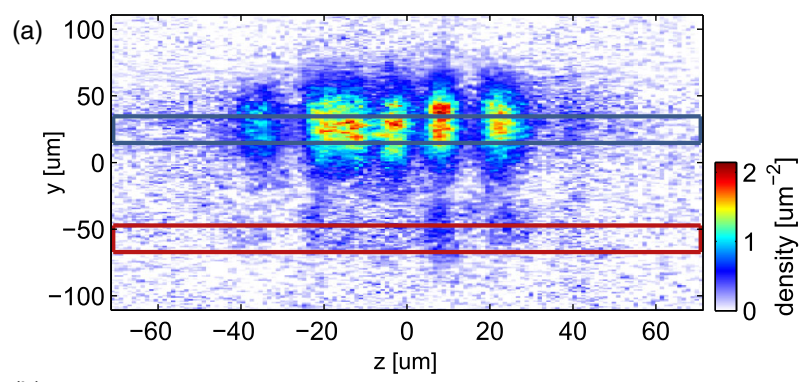

(b)

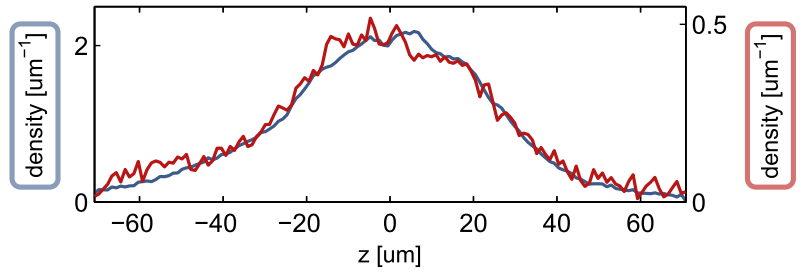

(c)

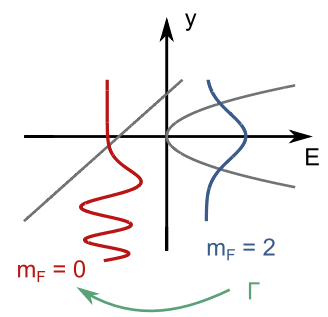

(d)

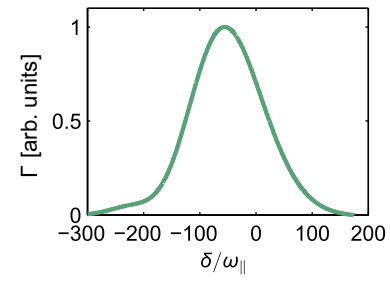

FIG. 1. Analysis of the outcoupling mechanism. (a) A typical density speckle pattern formed by a $1 \mathrm{D}$ quasicondensate expanding for $10.5 \mathrm{~ms}$ in time of flight. A small fraction was outcoupled by a single $2 \mathrm{~ms}$ rf pulse $2.5 \mathrm{~ms}$ before the gas was released. The outcoupled cloud is visible below the source cloud, showing the same speckle pattern. (b) Comparing the longitudinal density profiles of the source cloud (blue line) and the outcoupled cloud (red line), averaged over many realizations, reveals the same form of the profile. (c) Sketch of the outcoupling mechanism. Transversal to the $1 \mathrm{D}$ axis the atoms occupy the ground state of the harmonic potential (blue). Outcoupling is achieved by coupling these atoms to untrapped states, e.g., $|2,0\rangle$ (red), which only feel the linear gravitational potential. The rate of this transition, following from a simple calculation taking only the transversal degrees of freedom into account, is plotted in (d) over the detuning of the driving field, given in units of the longitudinal trap frequency. The large width of this rate compared to the energy scale of the longitudinal dynamics leads to a nearly homogeneous outcoupling of atoms.

1D axis [15]. To improve the individual images we employ a fringe removal algorithm [16]. The imaging provides access to the evolution of the atom number, the density profile, and the temperature of the gas. The latter is extracted from the longitudinal density speckle patterns forming in the time-of-flight expansion seen in Fig. 1(a) $[17,18]$. These patterns are a direct result of the longitudinal phase fluctuations in the trapped gas. Extracting their normalized autocorrelation function and comparing it to simulated data generated through a stochastic OrnsteinUhlenbeck process [19] allows us to infer the temperature of the gas.
Our results for the evolution of the atom number and the temperature for different initial conditions, outcoupling rates, and measurement procedures are shown in Fig. 2. The outcoupling is achieved through rf transitions within the $F=2$ manifold or microwave transitions to the antitrapped $|1,1\rangle$ state. We find that in all cases the correlation functions of the density speckles in time of flight remain close to their thermal form for all data points. Similarly, the relative phase fluctuations of two gases prepared in a double well potential through the same cooling mechanism also display an equilibrium form [20,21]. Such relative phase fluctuations have previously been shown to be very sensitive probes to athermal mode occupations [22]. These observations are remarkable, as the system is close to an integrable point and thus cannot thermalize on experimentally relevant time scales. In addition, the nearly homogeneous, energy-independent outcoupling process would intuitively not lead to cooling. Nevertheless, we observe a significant decrease in temperature down to $k_{B} T \sim 0.1 \hbar \omega_{\perp}$.

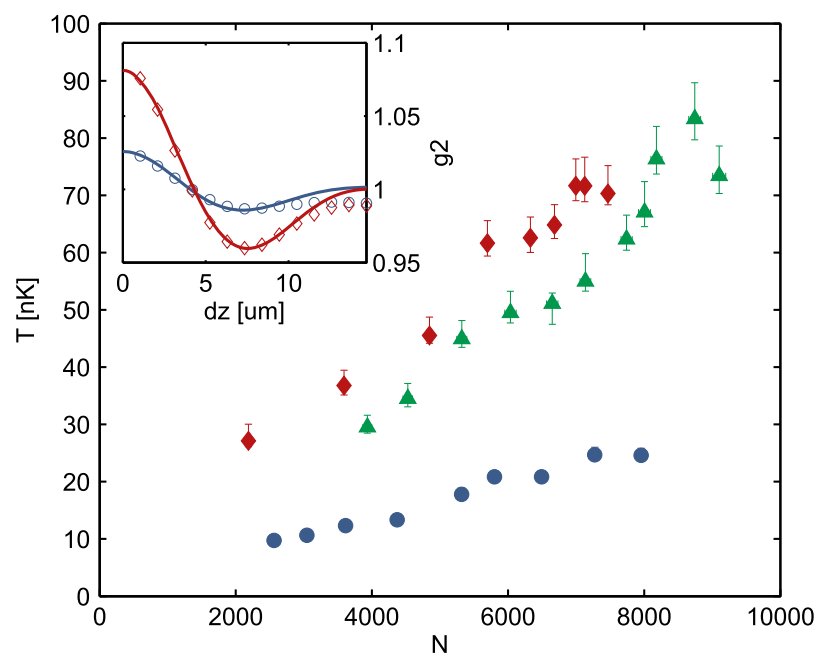

FIG. 2. Three different examples for measurements of temperature $T$ over atom number $N$. The green triangles are obtained through a continuous rf-induced outcoupling from a harmonic trap with trap frequencies $\omega_{\perp}=2 \pi \times(2.1 \pm 0.1) \mathrm{kHz}$ and $\omega_{\|}=$ $2 \pi \times(11 \pm 1) \mathrm{Hz}$ over a time scale of $25 \mathrm{~ms}$. The blue circles differ in that they start from a much colder initial temperature and were measured over a time scale of $90 \mathrm{~ms}$ in a transversally slightly anharmonic trap created through a dressed state potential [23]. There the trap frequencies are $\omega_{\perp}=2 \pi \times(1.6 \pm 0.1) \mathrm{kHz}$ and $\omega_{\|}=2 \pi \times(8 \pm 1) \mathrm{Hz}$. Finally, the red diamonds were acquired through a very different measurement procedure, where microwave radiation was used to couple atoms from the dressed trap directly to the antitrapped $|1,1\rangle$ state. Furthermore, in contrast to the other data points, the temperature was measured after a constant period of outcoupling with a different outcoupling rate for each data point. The temperature error bars give the $68 \%$ confidence interval obtained from a bootstrap. The inset depicts the speckle correlations of the coldest red and blue data points together with their respective thermal fits, still showing good agreement. 
Moreover, even though the system constantly loses atoms, the ratio $k_{B} T / \mu$ between the thermal energy and the interaction energy given by the chemical potential $\mu$ drops as well. In the coldest measurement presented in Fig. 2 (blue circles) it reaches values as low as $\sim 0.25$. The thermal coherence length $\lambda_{T}$ is inversely proportional to this ratio, showing that the system becomes more coherent under dissipation.

Figure 2 suggests that the temperature decreases linearly with the number of atoms. Rescaling the data points to their respective initial values $T_{0}$ and $N_{0}$ collapses the measurements to a single line, as shown in Fig. 3. This suggests a linear scaling relation between temperature and atom number,

$$
\frac{T}{T_{0}}=\frac{N}{N_{0}},
$$

which is particularly interesting as these measurements are obtained from different experimental procedures and initial conditions.

Model.- - In a pure 1D setting two-body collisions do not lead to a redistribution of energy and momentum. However, in a quasi-1D trap with a tight transverse confinement this condition can be broken in collisions where there is enough energy available to access transverse excited states. These thermalizing two-body collisions are suppressed by a factor $\exp \left(-2 \hbar \omega_{\perp} / k_{B} T\right)$ [7] in a gas of nondegenerate bosons. For a degenerate gas the Bose enhancement of the ground state leads to an even stronger suppression. Consequently, these collisions freeze out as soon as the gas enters the 1D regime. Other processes that can lead to thermalization in our system are three-body collisions $[7,8]$ or phononphonon scattering [9-11]. However, their expected thermalization time scales are beyond the times probed in our experiment and cannot explain the observed cooling. A different case of evaporative cooling in a 1D harmonic trap has been modeled by Witkowska et al. [24], assuming that atoms are outcoupled energy selectively at the two ends of

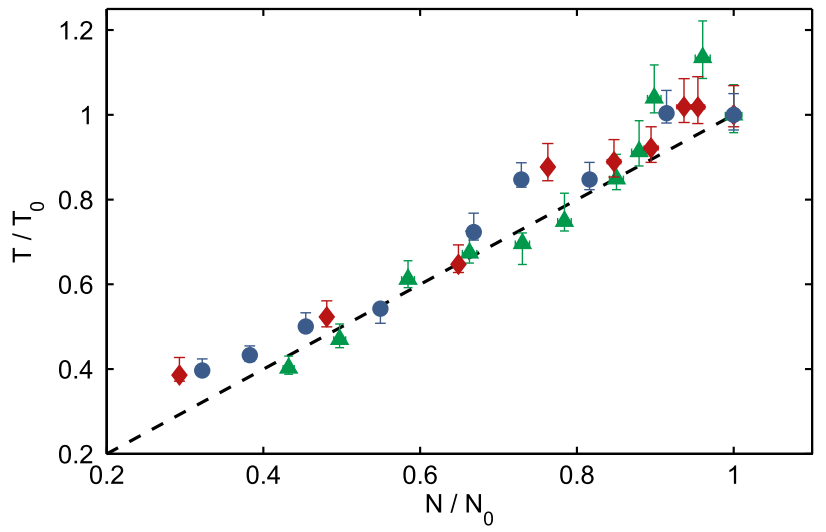

FIG. 3. The data from Fig. 2 are plotted rescaled to their respective initial temperature $T_{0}$ and atom number $N_{0}$ values revealing a clear linear scaling. the cigar-shaped trap, which is not the physical situation in our experiment (see Fig. 1).

To find a simple mechanism consistent with our experimental observations we start by considering the outcoupling process. The rate of outcoupling via if or microwave transitions is determined by the overlap of the condensate wave function with the free-particle states of the untrapped Zeeman levels [see Fig. 1(c)]. The observed homogeneity can be explained by the fact that the energy of the longitudinal degrees of freedom is not resolved by this process since the time scale on which atoms leave the trap is determined by the much tighter transversal confinement [see Fig. 1(d)]. This means that each atom has the same probability to leave the trap irrespective of its position or energy.

The dynamics resulting from such a homogeneous particle dissipation can be intuitively understood within a Luttinger-liquid picture [25], describing the low-energy dynamics of the underlying Lieb-Lininger model [26]. The elementary excitations in the Luttinger model are noninteracting phonon modes. Each of these modes contributes to the fluctuations in the gas through a density and a phase quadrature, in close analogy to the position and momentum quadratures of a harmonic oscillator. The free evolution of such a mode $k$ with energy $\hbar \omega_{k}$ can be visualized as a rotation of the corresponding Wigner function with the frequency $\omega_{k}$. In this picture, a sudden homogeneous outcoupling of atoms leads to a decrease in average density with the density fluctuations around this average being scaled down correspondingly. Such an instantaneous density reduction therefore extracts energy from the density quadrature of the phonon modes while leaving the phase quadrature unchanged. The system reacts to this reduction by dephasing, redistributing the remaining energy between the quadratures. A related process has recently been studied in detail using matter-wave interferometry after coherently splitting a 1D Bose gas and has been shown to lead to prethermalization [6,27] and light-cone-like spreading of thermal correlations [28].

The experimentally investigated situation of a slow, continuous dissipation can be modeled as a series of small density reductions $\rho(z) \rightarrow \rho^{\prime}(z)=\alpha \rho(z)$, each followed by an immediate dephasing. One such reduction casts the phonons in the system into a new basis, decreasing the mode occupations. For the experimentally relevant case of classical mode occupations $n_{k} \gg 1$, this process results in $n_{k} \rightarrow n_{k}^{\prime}=\frac{1}{2} \sqrt{\alpha}(\alpha+1) n_{k}$ (see Supplemental Material [29]). As the degenerate system is still sufficiently hot, the observable low-energy modes are dominated by the Rayleigh-Jeans part of the Bose-Einstein distribution where the mode occupation is proportional to the temperature $n_{k} \simeq k_{B} T / \epsilon_{k}$, with $\epsilon_{k}=\hbar c|k|$ being the mode energy. Here, $c=\sqrt{g \rho_{0} / m}$ denotes the speed of sound in the gas which depends on the average density $\rho_{0}$, the 1D interaction constant $g$, and the particle mass $m$. Since $c$ 
(a)

(b)
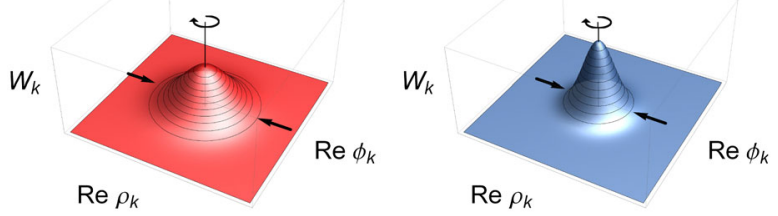

FIG. 4. Dissipative cooling mechanism visualized through the Wigner function $W_{k}$ (a) of the phase and density quadrature $\phi_{k}$ and $\rho_{k}$ of a single mode $k$, which rotates with the eigenfrequency $\omega_{k}$. The homogeneous outcoupling of atoms squeezes the Wigner function in the density quadrature, indicated by the black arrows, thereby reducing the energy of the mode. The rotation with $\omega_{k}$ together with a slow continuous squeezing leads to a (nearly) symmetric narrowing of the Wigner function (b) reflecting a reduced occupation of the mode. In our multimode system this happens in all modes at the same time, leading to a reduced temperature.

decreases with $\sqrt{\alpha}$ under particle dissipation, the temperature is reduced by the factor $\frac{1}{2} \alpha(\alpha+1)$, which, in the limit of infinitely many small reductions $\alpha \approx 1$ under continuous dephasing, results in the temperature scaling $T^{\prime} / T=\left(\rho_{0}{ }^{\prime} / \rho_{0}\right)^{3 / 2}$. Figure 4 illustrates this continuous decrease of the quasiparticle mode occupation through the Wigner function of a single phonon mode. In the case of a harmonically trapped gas in the Thomas-Fermi limit, the central density scales as $\rho_{0}^{\prime} / \rho_{0}=\left(N^{\prime} / N\right)^{2 / 3}$. Inserting this into the temperature scaling leads to the linear scaling relation $T^{\prime} / T=N^{\prime} / N$ observed in Fig. 3.

This result extends scaling laws for the dynamical compression and expansion of a trapped 1D Bose gas [30] to the realm of open quantum systems. Its universality is a result of the phononic nature of the excitations. Inhomogeneities in the outcoupling rate would not lead to a different scaling but to stable nonthermal energy distributions described by generalized Gibbs ensembles [22,31]. Supporting our simple model, the scaling law of Eq. (1) can also be found by numerically solving the Gross-Pitaevskii equation with an additional dissipative term. Furthermore, it is important to note that this cooling mechanism is similar to cooling by adiabatic expansion, in that the entropy per particle as well as the phase-space density stay constant.

It has been suggested that vacuum fluctuations enter the system through the dissipation process [32-34], which would lead to a reduced cooling efficiency. However, all of our measurements are consistent with the scaling of Eq. (1) derived from our simple model. This discrepancy represents an open question and is the topic of ongoing research.

Conclusion.-We have experimentally observed the cooling of a degenerate atomic gas in the $1 \mathrm{D}$ regime by continuous outcoupling of particles. In this process we reach temperatures as low as $k_{B} T \sim 0.25 \mu$ and $k_{B} T \sim 0.1 \hbar \omega_{\perp}$, which is far below the region where thermalizing two-body collisions freeze out, rendering standard evaporative cooling ineffective. The observed cooling allows us to go deep into the $1 \mathrm{D}$ regime and is therefore of direct practical importance for all experiments with 1D Bose gases. Our model also explains how previous experiments could observe such low temperatures $[35,36]$.

Finally, our simple model suggests that the mechanism behind the observed cooling is not limited to $1 \mathrm{D}$ and could be relevant in 2D and 3D settings at very low temperatures where atoms are homogeneously outcoupled from the quantum degenerate gas and the dephasing of excitations is faster than their thermalization.

We thank D. Adu Smith, M. Kuhnert, and M. Gring for contributions in the early stage of the experiment, and J. Walraven, A. Polkovnikov, E. Demler, and S. Weinfurtner for helpful discussions. This work was supported by the Austrian Science Fund (FWF) through the project P22590N16 and the SFB FoQuS Project F4010 and by the EU through the projects SIQS and the ERC advance grant QuantumRelax. B.R., P.G., and T.S. thank the FWF Doctoral Programme CoQuS (W1210). R. G. acknowledges support by the FWF through the Lise Meitner Programme M-1423. T. L. acknowledges support by the Alexander von Humboldt Foundation through a Feodor Lynen Research Fellowship.

*brauer@ati.ac.at

'Present address: Department of Quantum Matter Physics, University of Geneva, 24 Quai Ernest Ansermet, 1211 Geneva, Switzerland.

${ }^{\ddagger}$ Present address: SYRTE, Observatoire de Paris, 77 avenue Denfert Rochereau, 75014 Paris, France.

${ }^{\S}$ Present address: JILA, NIST and Department of Physics, University of Colorado, Boulder, Colorado 80309, USA. tlangen@ati.ac.at

[1] I. Bloch, J. Dalibard, and W. Zwerger, Rev. Mod. Phys. 80, 885 (2008).

[2] H. F. Hess, Phys. Rev. B 34, 3476 (1986).

[3] N. Masuhara, J. M. Doyle, J. C. Sandberg, D. Kleppner, T. J. Greytak, H. F. Hess, and G. P. Kochanski, Phys. Rev. Lett. 61, 935 (1988).

[4] O. J. Luiten, M. W. Reynolds, and J. T. M. Walraven, Phys. Rev. A 53, 381 (1996).

[5] T. Kinoshita, T. Wenger, and D. S. Weiss, Nature (London) 440, 900 (2006).

[6] M. Gring, M. Kuhnert, T. Langen, T. Kitagawa, B. Rauer, M. Schreitl, I. Mazets, D. A. Smith, E. Demler, and J. Schmiedmayer, Science 337, 1318 (2012).

[7] I. E. Mazets, T. Schumm, and J. Schmiedmayer, Phys. Rev. Lett. 100, 210403 (2008).

[8] S. Tan, M. Pustilnik, and L. I. Glazman, Phys. Rev. Lett. 105, 090404 (2010).

[9] A. Andreev, Sov. Phys. JETP 51, 1038 (1980).

[10] H.-P. Stimming, N. J. Mauser, J. Schmiedmayer, and I. E. Mazets, Phys. Rev. A 83, 023618 (2011).

[11] M. Buchhold and S. Diehl, Eur. Phys. J. D 69, 224 (2015). 
[12] J. Reichel and V. Vuletić, Atom Chips (Wiley-VCH, Weinheim, 2011).

[13] K. B. Davis, M.-O. Mewes, M. A. Joffe, M. R. Andrews, and W. Ketterle, Phys. Rev. Lett. 74, 5202 (1995).

[14] D. S. Petrov, G. V. Shlyapnikov, and J. T. M. Walraven, Phys. Rev. Lett. 85, 3745 (2000).

[15] D. A. Smith, S. Aigner, S. Hofferberth, M. Gring, M. Andersson, S. Wildermuth, P. Krüger, S. Schneider, T. Schumm, and J. Schmiedmayer, Opt. Express 19, 8471 (2011).

[16] C. F. Ockeloen, A. F. Tauschinsky, R. J. C. Spreeuw, and S. Whitlock, Phys. Rev. A 82, 061606 (2010).

[17] A. Imambekov, I. E. Mazets, D. S. Petrov, V. Gritsev, S. Manz, S. Hofferberth, T. Schumm, E. Demler, and J. Schmiedmayer, Phys. Rev. A 80, 033604 (2009).

[18] S. Manz, R. Bücker, T. Betz, C. Koller, S. Hofferberth, I. E. Mazets, A. Imambekov, E. Demler, A. Perrin, J. Schmiedmayer, and T. Schumm, Phys. Rev. A 81, 031610 (2010).

[19] H.-P. Stimming, N. J. Mauser, J. Schmiedmayer, and I. E. Mazets, Phys. Rev. Lett. 105, 015301 (2010).

[20] T. Betz, S. Manz, R. Bücker, T. Berrada, C. Koller, G. Kazakov, I. E. Mazets, H.-P. Stimming, A. Perrin, T. Schumm, and J. Schmiedmayer, Phys. Rev. Lett. 106, 020407 (2011).

[21] D. Adu Smith, M. Gring, T. Langen, M. Kuhnert, B. Rauer, R. Geiger, T. Kitagawa, I. Mazets, E. Demler, and J. Schmiedmayer, New J. Phys. 15, 075011 (2013).

[22] T. Langen, S. Erne, R. Geiger, B. Rauer, T. Schweigler, M. Kuhnert, W. Rohringer, I. E. Mazets, T. Gasenzer, and J. Schmiedmayer, Science 348, 207 (2015).
[23] T. Schumm, S. Hofferberth, L. M. Andersson, S. Wildermuth, S. Groth, I. Bar-Joseph, J. Schmiedmayer, and P. Krüger, Nat. Phys. 1, 57 (2005).

[24] E. Witkowska, P. Deuar, M. Gajda, and K. Rzążewski, Phys. Rev. Lett. 106, 135301 (2011).

[25] T. Giamarchi, Quantum Physics in One Dimension (Clarendon Press, Oxford, 2004).

[26] E. H. Lieb and W. Liniger, Phys. Rev. 130, 1605 (1963).

[27] M. Kuhnert, R. Geiger, T. Langen, M. Gring, B. Rauer, T. Kitagawa, E. Demler, D. Adu Smith, and J. Schmiedmayer, Phys. Rev. Lett. 110, 090405 (2013).

[28] T. Langen, R. Geiger, M. Kuhnert, B. Rauer, and J. Schmiedmayer, Nat. Phys. 9, 640 (2013).

[29] See Supplemental Material at http://link.aps.org/ supplemental/10.1103/PhysRevLett.116.030402 for a detailed derivation.

[30] W. Rohringer, D. Fischer, F. Steiner, I. E. Mazets, J. Schmiedmayer, and M. Trupke, Sci. Rep. 5, 9820 (2015).

[31] M. Rigol, V. Dunjko, V. Yurovsky, and M. Olshanii, Phys. Rev. Lett. 98, 050405 (2007).

[32] P. Grišins, B. Rauer, T. Langen, J. Schmiedmayer, and I. E. Mazets, arXiv:1411.4946.

[33] Y. Japha, S. Choi, K. Burnett, and Y. B. Band, Phys. Rev. Lett. 82, 1079 (1999).

[34] X. Busch, I. Carusotto, and R. Parentani, Phys. Rev. A 89, 043819 (2014).

[35] S. Hofferberth, I. Lesanovsky, T. Schumm, A. Imambekov, V. Gritsev, E. Demler, and J. Schmiedmayer, Nat. Phys. 4, 489 (2008).

[36] T. Jacqmin, J. Armijo, T. Berrada, K. V. Kheruntsyan, and I. Bouchoule, Phys. Rev. Lett. 106, 230405 (2011). 